\title{
Multiple-Symbol Differential Sphere Detection Aided Successive Relaying in the Cooperative DS-CDMA Uplink
}

\author{
Li Li and Lajos Hanzo \\ School of ECS, University of Southampton, SO17 1BJ, United Kingdom. \\ Tel: +44-23-8059 3125, Fax: +44-23-8059 4508 \\ Email: $\{115 \mathrm{e} 08, \mathrm{lh}\} @$ ecs.soton.ac.uk, http://www-mobile.ecs.soton.ac.uk
}

\begin{abstract}
The conventional amplify-and-forward cooperative system is capable of achieving a superior performance with the aid of MultipleSymbol Differential Sphere Detection (MSDSD), when compared to conventional differential detection (CDD) for transmission over timeselective channels. However, the conventional broadcast/cooperative twinphase based relaying protocol encounters a $50 \%$ throughput loss imposed by half-duplex relaying. For combating this problem, in this paper, we create a MSDSD aided successive relaying based cooperative DS-CDMA system. We demonstrate that given the target BER of $10^{-4}$, a diversity gain of up to $10 \mathrm{~dB}$ is achieved over the benchmark schemes employed without a throughput loss.
\end{abstract}

\section{INTRODUCTION}

Multiple-Input Multiple-Output (MIMO) techniques [1] are capable of providing a spatial diversity gain and hence efficiently mitigate the deleterious effects of hostile fading channels. However, the crucial condition for achieving a diversity gain is that the multiple co-located antenna elements are sufficiently far apart to experience independent fading. Regretfully, achieving transmit diversity in the uplink is typically impractical due to the limited size of the mobile. Fortunately, the family of cooperation techniques advocated by van der Meulen in [2] is capable of forming a virtual antenna array for each node (user) by allowing nodes (users) to relay the messages of others to the destination. Hence, such a relay aided network practically constitutes a distributed MIMO system, where cooperative diversity is achieved [3].

Most of the cooperative solutions found in the literature employ classic coherent detection. They also often assume the availability of perfect channel state information (CSI) at the receiver. However, practical pilot symbol assisted (PSA) techniques may impose a high pilot overhead, especially at high normalised Doppler frequencies. Furthermore, the channel estimation of a $M$-transmitter, $N$-receiver MIMO system requires the estimation of $(M \times N)$ CSIs, which significantly increases the entire system's complexity and consumes considerable extra energy, especially when the Doppler frequency is high. Hence the employment of non-coherent detection operating without any requirement of channel estimation becomes an attractive design alternative, especially in cooperative networks in the interest of reducing the relay's complexity. We note however that the performance of non-coherent receivers degrades substantially at high Doppler frequencies. Fortunately, this performance degradation may be efficiently mitigated with the aid of Multiple-Symbol Differential Detection (MSDD) at the cost of an increased complexity. We then invoke sophisticated sphere detection to mitigate the complexity imposed.

The MSDD concept was proposed by Van Trees in [4], while its decision matrix and decision criterion were detailed by Ho and Fung in [5]. Furthermore, Lampe et al. devised the MultipleSymbol Differential Sphere Detection (MSDSD) algorithm in [6] by combining sphere decoding with MSDD based on Ho's modified decision matrix. It was demonstrated in [6] that MSDSD substantially reduces the complexity of MSDD and consequently becomes practical. Recently, the MSDSD algorithm was also advocated in the

The financial support of the European Union under the auspices of the Newcom and Phoenix projects, as well as that of the RC-UK under the auspices of the India-UK Advanced Technology Centre known as IN-ATC is gratefully acknowledged. context of cooperative communication systems [7] [8], and the singleuser MSDSD algorithm was further developed for employment in single-user multiple-path scenarios.

However, the conventional two phase cooperative regime incurs a significant multiplexing loss due to the half-duplex constraint of practical transceivers. Inspired by the results of [7], our main contributions in this paper are

1) We further develop the single-user/single-path MSDSD algorithm of [6] into a novel relay-aided form. More specifically, we will demonstrate that compared to the multiple-path MSDSD algorithm of [7, (26)], the size of the matrix $\mathbf{U}$ of our novel relay-aided MSDSD only increases linearly with the number of entities (source node plus relays) and hence the system complexity is reduced.

2) We then incorporate our relay-aided MSDSD algorithm in a novel successive relaying assisted cooperative network, which achieves a similar performance gain to the system of [7], while additionally recovering the half-duplex relaying induced throughput loss.

3) We effectively reduce the successive relaying induced interference between the transmitted signals of the source and relay with the aid of classic DS-CDMA principles.

The rest of this paper is organised as follows. Our system model is described in Section II. Section III details our novel scheme designed for executing the MSDSD algorithm, which is termed here as the distributed relay-aided MSDSD. Then Section IV analyses the requirement of the specific coding and decoding strategy. Our simulation results and their discussions are provided in Section V. Finally, we conclude in Section VI.

\section{System OVERVIEW}

We consider the uplink of the relay assisted DS-CDMA system of Figure 1, where $M S_{n}$ and $h_{s_{n} d}, n=1,2, \ldots, M$, represent the Mobile Stations (MSs) and the Channel Impulse Responses (CIR) spanning from the source to the destination, respectively. We assume that some of the idle MSs are willing to act as relays and they exclusively assist one of the $M$ users seen at the left hand side of Figure 1 based on the successive relaying protocol of [9]. Explicitly, the elements of Figure 1 constitute a classic successive relaying aided cooperative network using DS-CDMA transmissions. Let us now focus our attention on this successive relaying regime. Figure 2 further details the principles portrayed in Figure 1, displaying three scenarios corresponding to the different cooperative frames. We assume that the two relays are sufficiently far apart, so that the channels experienced by the different relays experience reasonably independent fading with respect to each other even in the presence of correlated shadowing and hence achieve the maximum attainable spatial diversity. The interference between two relays is neglected in this paper, in other words relay-a is assumed to receive no interference from relay-b and vice versa. We also assume that the signals are perfectly synchronised, for example using the solution of [10] and that perfect power control is available, hence we can neglect the pass-loss effects. To improve the practicability of this contribution, classic frame-by-frame based transmissions routinely employed in realistic communication networks are considered here. According to 


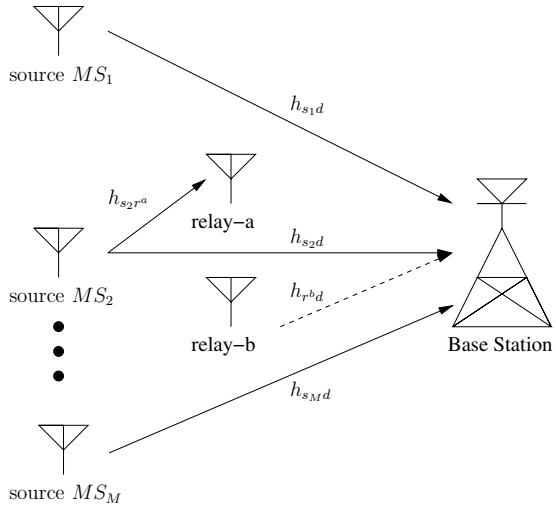

Fig. 1. The simplified uplink diagram of the successive relay-assisted DSCDMA system, assuming that the successive relaying aided cooperative subsystem is embedded in the middle.

the specific structure of the successive relaying aided cooperative network, the frame-based transmission regime delivers a specific codeword which is forwarded by the Relay Node $(\mathrm{RN})$ and will arrive at the Destination Node (DN) a frame period later with respect to the directly received replica transmitted by the Source Node (SN). Hence the system delay is related to the frame length $L$. Based on these assumptions, Figure 2 summarizes the main properties of the successive relaying aided network.


Fig. 2. The successive relaying aided cooperative sub-system, during various cooperative frames.

As detailed in [11], the most important feature of successive relaying aided cooperative networks is that the signals directly transmitted by the SN and the signals forwarded by one of the two RNs are always simultaneously received at the DN. Provided that this condition was satisfied, the algorithm discussed in [12] provides a feasible method of extracting the different components from the resultant composite signal with an acceptable accuracy. Inspired by [12], the DS-CDMA technique was adopted in order to avoid the potential interference between the transmitted signals of the SN and $\mathrm{RN}$, which was further developed for our successive relaying aided network. In more detail, first the $(L-1)$ information symbols $x[k]$ $\in \chi=\left\{e^{j 2 \pi m / M} ; m=0,1, \cdots, M-1\right\}$ involved in the $l$ th frame are differentially encoded at the $\mathrm{SN}$ and consequently generate $L$ MDPSK modulated signals, as follows [7]

$$
\begin{aligned}
s[k] & =\sqrt{P_{s}} \cdot s[k-1] \cdot x[k-1] \\
& =\sqrt{P_{s}} \cdot e^{j 2 \pi m / M}, m=0,1, \cdots, M-1 .
\end{aligned}
$$

where the index $k$ indicates the $n$th MDPSK signal in the $l$ th frame, which is specified as $k=l \cdot L+n$. We divide all the frames into two sets, according to which specific RN receiver was activated during that frame. The frame in which relay-a is receiving is referred to as frame $\mathrm{A}$ and the frame during which relay-b is receiving becomes frame B. For example, frame 2 and frame 3 depicted in Fig. 2 correspond to frame-set B and frame-set A, respectively. Then, a specific DS-CDMA spreading strategy is implemented: two different binary DS spreading waveforms, namely $C_{A}(t), C_{B}(t)$ chosen from the entire spreading sequence family are employed for spreading the signals transmitted by the $\mathrm{SN}$ during frame $\mathrm{A}$ and frame $\mathrm{B}$, respectively.
Furthermore, the amplify-and-forward relaying scheme [8] is adopted in order to impose a lower complexity compared to that of the decode-and-forward scheme [8]. For the sake of a fair comparison with classic direct communications using no relays, the overall transmitted power should be constrained to unity. Hence, if $P_{s}$ and $P_{r} u$ are defined as the power assigned to the $\mathrm{SN}$ and to the $u$ th $\mathrm{RN}$, respectively, the total power required by the $U$ entities (SN plus RNs) can be expressed as $\Sigma_{u=1}^{U-1} P_{r u}=1-P_{s}$. In our scenario we have $U=3$. According to [7] and to the analysis of the delay associated with the relayed signals, the amplified signal forwarded by the $u$ th $\mathrm{RN}$ may be expressed as

$$
\begin{aligned}
s_{r^{u} d}[k] & =f_{A M_{r u} u} y_{s r^{u}}[k-L] \\
& =f_{A M_{r^{u}}}\left\{s_{s d}[k-L] h_{s r^{u}}[k-L]+n_{s r^{u}}[k-L]\right\},
\end{aligned}
$$

where $y_{s r^{u}}[k-L]$ represents the source-to-relay signal received at the $u$ th RN during the last frame; $s_{s d}[k-L]$ is the signal broadcast from the $\mathrm{SN}$ in the last frame; $h_{s r} u[k-L]$ corresponds to the CIR between the $\mathrm{SN}$ and the $u$ th $\mathrm{RN}$ in the last frame; $n_{s r^{u}}[k-L]$ is the AWGN noise; $f_{A M_{r} u}$ is the amplification factor of the $u$ th relay, which should ensure that the average transmit power of the $u$ th relay equals to $P_{r^{u}}$, and is given by [13]

$$
f_{A M_{r} u}=\sqrt{\frac{P_{r^{u}}}{P_{s} \sigma_{s r^{u}}^{2}+N_{0}}},
$$

where $\sigma_{s r u}^{2}$ represents the variance of the channel's complex envelope between the $\mathrm{SN}$ and the $u$ th $\mathrm{RN}$, while $N_{0}$ is the noise variance.

Let us now define

$$
\begin{aligned}
\mathbf{Y}_{A} & =\left[y_{A(0)}, y_{A(1)}, \cdots, y_{A(Q-1)}\right]^{T} \\
\mathbf{Y}_{B} & =\left[y_{B(0)}, y_{B(1)}, \cdots, y_{B(Q-1)}\right]^{T} \\
\mathbf{C}_{A} & =\frac{1}{\sqrt{Q}}\left[c_{A(0)}, c_{A(1)}, \cdots, c_{A(Q-1)}\right]^{T} \\
\mathbf{C}_{B} & =\frac{1}{\sqrt{Q}}\left[c_{B(0)}, c_{B(1)}, \cdots, c_{B(Q-1)}\right]^{T},
\end{aligned}
$$

where $y_{A(q)}$ represents the received signal chip A at the DN in a chip-duration and all the A-chips constitute vector $\mathbf{Y}_{A}$, which represents the signal vector corresponding to the entire symbolduration. The variables $y_{B(q)}$ and $\mathbf{Y}_{B}$ have similar definitions and the same relationship with respect to $y_{A(q)}$ and $\mathbf{Y}_{A}$. Explicitly, $c_{A(q)}$ or $c_{B(q)}$ is one of the chip-waveforms and belongs to $\mathbf{C}_{A}$ or $\mathbf{C}_{B}$, whose spreading factor is $Q$. Hence, the exact components of the signal received at the $\mathrm{DN}$ in the $\mathrm{A}$ or $\mathrm{B}$ type frames may be formulated respectively as

$$
\begin{aligned}
& \mathbf{Y}_{A}[k]=s[k] \mathbf{C}_{A} h_{s d}[k]+n_{d}[k]+ \\
& \quad f_{A M_{r b} b}\left\{s[k-L] \mathbf{C}_{B} h_{s r^{b}}[k-L]+n_{s r^{b}}[k-L]\right\} h_{r^{b} d}[k] \\
& \mathbf{Y}_{B}[k]=s[k] \mathbf{C}_{B} h_{s d}[k]+n_{d}[k]+ \\
& f_{A M_{r^{a}} a}\left\{s[k-L] \mathbf{C}_{A} h_{s r^{a}}[k-L]+n_{s r^{a}}[k-L]\right\} h_{r^{a} d}[k] .
\end{aligned}
$$

The specific DS-CDMA spreading strategy employed enables us to alternatively despread the signals received at the DN by reconfiguring the chip-waveform matched-filter according to the frame type (A or $\mathrm{B}$ ). In the $l$ th frame $\mathrm{A}$, we first employ a filter matched to the waveform of $\mathbf{C}_{A}$. The main component of the despread signal is the source signal $s[k]$, while the component corresponding to the source signal $s[k-L]$ forwarded by relay-b becomes the interference. At the next step, we reconfigure the filter to match the waveform of $\mathbf{C}_{B}$. Consequently, the main component becomes the previous source signal $s[k-L]$, and simultaneously the current source signal $s[k]$ acts as the interference. The pair of different despread signals generated from the same received signal during frame $\mathrm{A}$ is described by

$$
z_{A}^{s{ }^{\prime}}[k]=s[k] h_{s d}[k]+I_{r^{b}}[k]+N_{s r^{b}}^{\prime}[k]+N_{d}[k]
$$


and

$$
\begin{aligned}
z_{A}^{r^{b^{\prime}}}[k]= & s[k-L] h_{s r^{b}}[k-L] f_{A M_{r^{b}}} h_{r^{b} d}[k]+I_{s}[k]+ \\
& N_{s r^{b}}^{\prime}[k]+N_{d}[k],
\end{aligned}
$$

where both $I_{r^{b}}[k]=\frac{1}{Q} s[k-L] h_{s r^{b}}[k-L] f_{A M_{r^{b}}} h_{r^{b} d}[k]$ and $I_{s}[k]=\frac{1}{Q} s[k] h_{s d}[k]$ represent the interfering components. Then the AWGN imposed on the signals received by relay-b will be amplified by a factor of $f_{A M_{r b}}$ and transmitted via the channel between relay-b and the DN. Finally, we arrive at $N_{s r^{b}}^{\prime}[k]=$ $\mathbf{C}_{A}^{T} n_{s r^{b}}[k-L] f_{A M_{r b}} h_{r^{b} d}[k]$, which can be rewritten as $N_{s r^{b}}^{\prime}[k]=$ $n_{s r^{b}}[k-L] f_{A M_{r b}} h_{r^{b} d}^{r^{b}}[k]$ and has an equivalent effect on the system. Furthermore, $N_{d}[k]=\mathbf{C}_{A}^{T} n_{d}[k]$ is the AWGN imposed on the signal received at the $\mathrm{DN}$, which can also be rewritten as $N_{d}[k]=n_{d}[k]$ for convenience. Similarly, in frame B, two differently despread signals can be obtained, which are represented by

$$
z_{B}^{s}{ }^{\prime}[k]=s[k] h_{s d}[k]+I_{r^{a}}[k]+N_{s r^{a}}^{\prime}[k]+N_{d}[k]
$$

and

$$
\begin{aligned}
z_{B}^{r^{a \prime}}[k]= & s[k-L] h_{s r^{a}}[k-L] f_{A M_{r^{a}}} h_{r^{a} d}[k]+I_{s}[k]+ \\
& N_{s r^{a}}^{\prime}[k]+N_{d}[k],
\end{aligned}
$$

where the terms such as $I_{r^{a}}[k], I_{s}[k], N_{s r^{a}}^{\prime}[k]$, and $N_{d}[k]$ maintain similar properties to those mentioned before. In order to benefit from the particular spreading and despreading strategies employed, the interference imposed by successive relaying can also be successfully mitigated, when employing an amplify-and-forward relaying scheme. More explicitly, we are not constrained to the employment of decode-and-forward relaying schemes, when considering the interrelay interference cancellation problem in the context of cooperative systems. Hence the DS-CDMA despread, decode, re-encode and respread operations employed at the RN in [12] are avoided, therefore, the system's complexity is reduced. Additionally, the number of spreading sequences required is also lower compared to [12].

\section{Principle of Relay-Aided Multiple-Symbol DifFERENTIAL SPHERE DETECTION}

The MSDSD algorithm was advocated in the context of cooperative communication systems in [7]. One of the critical design issues is that of combining the multiple relay-aided received signals, which correspond to the same source symbols but are transmitted by multiple users and experience different channels. Clearly, we want to generate a single observed signal sequence, similar to that of the single-user MSDSD operations. Consequently, the single-user MSDSD may be directly applied to process this composite signal sequence. The extension of the single-user MSDSD to the multiplepath MSDSD of [7] is feasible, but the size of the critical matrix $\mathbf{U}$ of $[7,(26)]$ rapidly increases and the implementation of sphere detection based on this matrix becomes a challenge, when the number of users increases. Hence, a new relay-aided MSDSD concept is introduced here. Without loss of generality, let us define

$$
\begin{aligned}
\mathbf{X} & =[x[1], x[2], \cdots, x[N-1]]^{T} \\
\mathbf{S} & =[s[1], s[2], \cdots, s[N]]^{T} \\
\mathbf{Z}^{u} & =\left[z^{u}[1], z^{u}[2], \cdots, z^{u}[N]\right]^{T} \\
\mathbf{H}^{u} & =\left[h^{u}[1], h^{u}[2], \cdots, h^{u}[N]\right]^{T},
\end{aligned}
$$

where $\mathbf{X}$ is the original MPSK information symbol sequence and $\mathbf{S}$ is the differentially encoded MDPSK symbol sequence. Furthermore, $\mathbf{Z}^{u}$ is the individual symbol sequence received from the $u$ th entity ( $\mathrm{SN}$ or RN). Finally, $\mathbf{H}^{u}$ is the CIR vector of the channel between the $u$ th entity and the DN, while $N$ is the observation window size of MSDSD, which is a time domain parameter.

Similar to the situation discussed in [14], provided that the assumptions made in Section II are satisfied, the received sequences
$\left\{\mathbf{Z}^{u}\right\}_{u=1,2, \cdots, U}$ are independent Gaussian random vectors. Hence the corresponding conditional probability density function can be written as

$$
p\left(\left\{\mathbf{Z}^{u}\right\}_{u=1,2, \cdots, U} \mid \mathbf{S}\right)=\prod_{u=1}^{U} p\left(\mathbf{Z}^{u} \mid \mathbf{S}\right),
$$

where $U$ is the number of entities involved in cooperative communication. According to [5], when an MDPSK sequence was transmitted, the conditional probability density function of the $u$ th received sequence $\mathbf{Z}^{u}$ is represented as

$$
\begin{aligned}
p\left(\mathbf{Z}^{u} \mid \mathbf{S}\right) & =\frac{1}{(2 \pi)^{\frac{N}{2}} \| \Phi_{\mathbf{Z}^{u} \mathbf{Z}^{u} \|^{\frac{1}{2}}}} \exp \left(-\frac{1}{2} \mathbf{Z}^{u H} \boldsymbol{\Phi}_{\mathbf{Z}^{u} \mathbf{Z}^{u}}^{-1} \mathbf{Z}^{u}\right) \\
& =\eta_{u} \cdot \exp \left(-\frac{1}{2} \mathbf{Z}^{u H} \boldsymbol{\Phi}_{\mathbf{Z}^{u} \mathbf{Z}^{u}}^{-1} \mathbf{Z}^{u}\right) .
\end{aligned}
$$

Upon substituting (13) in (12), (12) may be rewritten as

$$
p\left(\left\{\mathbf{Z}^{u}\right\}_{u=1,2, \cdots, U} \mid \mathbf{S}\right)=\left(\prod_{u=1}^{U} \eta_{u}\right) \times \exp \left(-\frac{1}{2} \sum_{u=1}^{U} \mathbf{Z}^{u H} \mathbf{\Phi}_{\mathbf{Z}^{u} \mathbf{Z}^{u}}^{-1} \mathbf{Z}^{u}\right),
$$

where the factor $\prod_{u=1}^{U} \eta_{u}$ is independent of $\mathbf{S}$. Hence, the relayaided MSDSD decision rule of finding the specific sequence $\mathbf{S}^{\prime}=$ $\left[s^{\prime}[1], s^{\prime}[2], \cdots, s^{\prime}[N]\right]^{T}$ which maximises the conditional probability density function of (14) is equivalent to selecting the particular transmitted source signal sequence $\mathbf{S}^{\prime}$, whose matrix sum is the smallest, which is formulated as

$$
\mathbf{S}^{\prime}=\underset{\mathbf{S}}{\arg \min }\left\{\sum_{u=1}^{U} \mathbf{Z}^{u H} \boldsymbol{\Phi}_{\mathbf{Z}^{u} \mathbf{Z}^{u}}^{-1} \mathbf{Z}^{u}\right\}
$$

where the correlation matrix obeys: $\mathbf{\Phi}_{\mathbf{Z}^{u} \mathbf{Z}^{u}} \triangleq \operatorname{diag}\{\mathbf{S}\} \mathbf{C}^{u} \operatorname{diag}\left\{\mathbf{S}^{*}\right\}$ and $\mathbf{C}^{u} \triangleq \mathcal{E}\left\{\mathbf{H}^{u} \mathbf{H}^{u H}\right\}+\sigma_{n}^{2} \mathbf{I}_{N}$.

Similar to [6], the new relay-aided MSDSD decision rule can be rewritten as

$$
\mathbf{S}^{\prime}=\underset{\mathbf{S}}{\arg \min }\left\{\sum_{u=1}^{U}\left\|\mathbf{U}^{u} \mathbf{S}\right\|^{2}\right\}
$$

where we have $\mathbf{U}^{u} \triangleq\left(\left(\mathbf{L}^{u}\right)^{H} \operatorname{diag}\left\{\mathbf{Z}^{u}\right\}\right)^{*}$, which is an upper triangular matrix. Then the corresponding lower triangular matrix $\mathbf{L}^{u}$ is defined by the Cholesky factorization of the inverse of the matrix $\mathbf{C}^{u}$, namely of $\left(\mathbf{C}^{u}\right)^{-1}=\mathbf{L}^{u}\left(\mathbf{L}^{u}\right)^{H}$. The more detailed version of the matrix $\mathbf{U}^{u}$ is formulated as

$$
\mathbf{U}^{u}=\left[\begin{array}{cccc}
\mathbf{U}_{1,1}^{u} & \mathbf{U}_{1,2}^{u} & \cdots & \mathbf{U}_{1, N}^{u} \\
0 & \mathbf{U}_{2,2}^{u} & \cdots & \mathbf{U}_{2, N}^{u} \\
\vdots & \vdots & \ddots & \vdots \\
0 & 0 & \cdots & \mathbf{U}_{N, N}^{u}
\end{array}\right]_{N \times N}
$$

Then, we further define a $(U N \times N)$-element matrix $\mathbf{U}$ as

$$
\mathbf{U}=\left[\begin{array}{cccc}
\mathbf{U}_{1,1} & \mathbf{U}_{1,2} & \cdots & \mathbf{U}_{1, N} \\
0 & \mathbf{U}_{2,2} & \cdots & \mathbf{U}_{2, N} \\
\vdots & \vdots & \ddots & \vdots \\
0 & 0 & \cdots & \mathbf{U}_{N, N}
\end{array}\right]_{U N \times N}
$$

and

$$
\mathbf{U}_{i, j}=\left[\mathbf{U}_{i, j}^{1}, \mathbf{U}_{i, j}^{2}, \cdots, \mathbf{U}_{i, j}^{U}\right]^{T},
$$

where $\mathbf{U}_{i, j}$ is the specific vector component of $\mathbf{U}$ in row $i$ and column $j, 1 \leq i, j \leq N, \mathbf{U}_{i, j}^{u}$ denotes the entry of $\mathbf{U}^{u}$ found in row $i$ and column $j$. Upon substituting (18) into (16), we finally arrive at our relay-aided MSDSD decision rule

$$
\mathbf{S}^{\prime}=\underset{\mathbf{S}}{\arg \min }\left\{\|\mathbf{U S}\|^{2}\right\} \text {. }
$$


Compared to the single-user/single-stream MSDSD of [6], to implement the proposed relay-aided MSDSD given by (20), the difference manifests itself in the definition of the best candidate point $s_{i}^{\prime}$ for the $i$ th estimated symbol in $\mathbf{S}^{\prime}$, which has to satisfy

$$
s_{i}^{\prime}=\underset{\chi}{\arg \min }\left\{\sum_{u=1}^{U}\left|\mathbf{U}_{i, i}^{u} s_{i}^{\prime}+\sum_{j=i+1}^{N} \mathbf{U}_{i, j}^{u} s_{j}^{\prime}\right|\right\} .
$$

The size of the corresponding matrix $\mathbf{U}$ used in [7, (26)] is $\left(U N \times U^{2} N\right)$, which is cubically proportional to the number of entities $U$. By contrast, the size of the newly devised matrix $\mathbf{U}$ of (18) is reduced to $(U N \times N)$, which only increases linearly with $U$. Furthermore, in contrast to the channel-noise autocorrelation matrix $\mathbf{C}^{u}$ involved in (15), which is computed only once during the entire detection process, the calculation of the matrix $\mathbf{U}$ is repeated in every observation window. Hence our relay-aided MSDSD proposed in this paper reduces the complexity imposed, especially for $U>3$.

Our algorithm may be decomposed into two steps: first, we individually calculate each $\mathbf{U}^{u}$ contribution seen in (17) according to the conventional single-user MSDSD algorithm; then, we execute the relay-aided MSDSD based on the matrix $\mathbf{U}$ of (18), which is constituted by combining all the $\mathbf{U}^{u}$ s components based on (19).

\section{RELAY-Aided MSDSD DESIGN FOR SuCCESSIVE RELAYING BASED COOPERATION}

Our motivation in this section is to incorporate the relay-aided MSDSD of Section III in the successive relay-aided cooperative regime of Figure 2, and consequently create the relay-aided MSDSD assisted successive relaying based cooperative DS-CDMA system. Without loss of generality, first we observe a length- $N$ normalized MDPSK symbol block broadcast by the SN during frame A, namely $\mathbf{S}$, which is represented as

$$
\mathbf{S}=\left[\frac{1}{\sqrt{P_{s}}} s[k+1], \frac{1}{\sqrt{P_{s}}} s[k+2], \cdots, \frac{1}{\sqrt{P_{s}}} s[k+N]\right]^{T} .
$$

According to the analysis provided in Section II, one of the two replicas of the observed MDPSK symbol block $\mathbf{S}$ is generated by the source-to-destination transmission in the same frame of $\mathbf{S}$ and actually constituted by the despread signals $z_{A}^{s}{ }^{\prime}[k]$ of (7) as

$$
\mathbf{Z}_{A}^{s}=\left[z_{A}^{s{ }^{\prime}}[k+1], z_{A}^{s \prime}[k+2], \cdots, z_{A}^{s{ }^{\prime}}[k+N]\right]^{T} .
$$

The another replica of the observed MDPSK symbol block $\mathbf{S}$ forwarded by relay-a will arrive at the DN $L$ symbol periods later in the next frame and actually be constituted by the despread signals $z_{B}^{r^{a \prime}}[k]$ of $(10)$ as

$$
\mathbf{Z}_{B}^{r^{a}}=\left[z_{B}^{r^{a \prime}}[k+L+1], \cdots, z_{B}^{r^{a \prime}}[k+L+N]\right]^{T} .
$$

Clearly, according to the principle described in Section III, we can directly implement the relay-aided MSDSD algorithm by utilizing $\mathbf{Z}_{A}^{s}$ and $\mathbf{Z}_{B}^{r^{a}}$ as the $\mathbf{Z}^{u}$ components in (12). The entire process is illustrated in more detail by Figure 3, where the exact activities of each entity in the relay-aided MSDSD assisted successive relaying based cooperative network are portrayed during frame A or B.

Furthermore, the vector $\mathbf{Z}_{A}^{s}$ in (23) may be rewritten in a more compact matrix form as

$$
\mathbf{Z}_{A}^{s}=\mathbf{H}_{A}^{s} \mathbf{S}+\mathbf{W}_{A}^{s},
$$

where $\mathbf{H}_{A}^{s}$ and $\mathbf{W}_{A}^{s}$ are given by (26) and (27), respectively, which are the equivalent CIR matrix and the equivalent noise matrix, respectively.

$$
\mathbf{H}_{A}^{s}=\left[\begin{array}{c}
\sqrt{P_{s}} h_{s d}[k+1] \\
\sqrt{P_{s}} h_{s d}[k+2] \\
\vdots \\
\sqrt{P_{s}} h_{s d}[k+N]
\end{array}\right]_{N \times 1}
$$

Here, the interference component $I_{r b}$ is approximated by a noise process, hence the equivalent noise process of (27) is constituted by the 2nd, 3rd and 4th components of (7), which are the interference term $I_{r^{b}}$, the amplified and faded noise component $N_{s r^{b}}^{\prime}$ and the AWGN $N_{d}$. Furthermore, (28) (29) are provided to facilitate the calculation of $\mathbf{U}^{s}$ of (17) which corresponds to $\mathbf{Z}_{A}^{s}$.

$\mathcal{E}\left\{\mathbf{H}_{A}^{s} \mathbf{H}_{A}^{s}{ }^{H}\right\}=P_{s} \times$

$\left[\begin{array}{cccc}\varphi_{s d}^{t}[0] & \varphi_{s d}^{t}[1] & \cdots & \varphi_{s d}^{t}[N-1] \\ \varphi_{s d}^{t}[-1] & \varphi_{s d}^{t}[0] & \cdots & \varphi_{s d}^{t}[N-2] \\ \vdots & \vdots & \ddots & \vdots \\ \varphi_{s d}^{t}[1-N] & \varphi_{s d}^{t}[2-N] & \cdots & \varphi_{s d}^{t}[0]\end{array}\right]_{N \times N}$

$\mathcal{E}\left\{\mathbf{W}_{A}^{s} \mathbf{W}_{A}^{s H}\right\}=\mathbf{I}_{N \times N} \times$

$\left(\frac{1}{Q^{2}} P_{s} \sigma_{s r^{b}}^{2} f_{A M_{r b} b}^{2} \sigma_{r^{b} d}^{2}+\left(\sigma_{r^{b} d}^{2} f_{A M_{r^{b}}}^{2}+1\right) N_{0}\right)$.

Then, the vector $\mathbf{Z}_{B}^{r^{a}}$ in (24) obeys a similar relationship to $\mathbf{Z}_{A}^{s}$, yielding

$$
\mathbf{Z}_{B}^{r^{a}}=\mathbf{H}_{B}^{r^{a}} \mathbf{S}+\mathbf{W}_{B}^{r^{a}},
$$

where the $\mathbf{H}_{B}^{r^{a}}$ and $\mathbf{W}_{B}^{r^{a}}$ are similar to that conceived for $\mathbf{H}_{A}^{s}$ and $\mathbf{W}_{A}^{s}$, respectively. Furthermore, the $\mathbf{U}^{r^{a}}$ corresponding to $\mathbf{Z}_{B}^{r^{a}}$ can be attained in a similar way to the calculation of $\mathbf{U}^{s}$.

According to the principles outlined in Section III, after individually calculating the matrices $\mathbf{U}^{s}$ and $\mathbf{U}^{r^{a}}$, we combine them to create the matrix $\mathbf{U}$ as detailed in (18) and (19). Finally, we invoke sphere detection according to (20) and (21), in order to complete the entire detection process of $\mathbf{S}$.

Based on the symmetry of the transmission mechanisms between frame $\mathrm{A}$ and $\mathrm{B}$, the decoding process of the normalized MDPSK symbol blocks broadcast by the SN during frame B is similar to that conceived for $\mathbf{S}$, as detailed above.

\section{Simulation Results}

Based on the analysis outlined in the previous sections, compared to the conventional two-phase Amplify-and-Forward (AF) cooperative procedure operations with the aid of the multiple-path MSDSD algorithm of [7], the new scheme proposed in this paper recovers the factor-two throughput loss. However, this throughput improvement is achieved at the cost of imposing inter-relay interference plus the amplified noise $N_{s r^{b}}{ }^{\prime}$ of (7) on the received signals, which may erode the performance gain. Hence, the simulation results of Figure 4 characterize the trade-offs between the achievable throughput and BER performance attained. In Figure 4, a time-selective communication environment is assumed, where the normalized Doppler Frequency (DF) is fixed to 0.01 for all experiments based on our proposed systems. Then, the observation window size of MSDSD is fixed to $N=6$ and DQPSK modulation is employed. The interpretations of the benchmark schemes employed for comparison are given in Table I. Furthermore, due to the $50 \%$ throughput loss effect, the normalized Doppler frequency of benchmark-III is adjusted to 0.02 for fair comparison. The interference between the transmitted signals of the SN and RN imposed by the successive relaying protocol significantly affects the system's performance. As shown in Figure 4, increasing the DS-CDMA Spreading Factor (SF) $Q$ mitigates the influence of interference. Nevertheless, an error floor is encountered for a SF of 32 between $\mathrm{BER}=10^{-4}$ and $10^{-5}$. No significant BER improvement can be attained upon increasing the SF beyond 64, but nevertheless a SF of 512 will be adopted in our forthcoming investigations for minimizing the influence of interference in adverse propagation conditions, as justified at a later stage in the context of Figure 5.

The relay-aided MSDSD assisted successive relaying system using $\mathrm{SF}=512$ requires an approximately $1 \mathrm{~dB}$ higher transmit power to achieve $\mathrm{BER}=10^{-4}$ compared to its single-relay aided conventional 
Jointly detect $[s[k+1], s[k+2], \cdots, s[k+N]]$ using relay-aided MSDSD

Fig. 3. Frame structure of the relay-aided MSDSD assisted successive relaying based cooperative system, where the solid box represents the transmitted signals, the dashed box the received signals

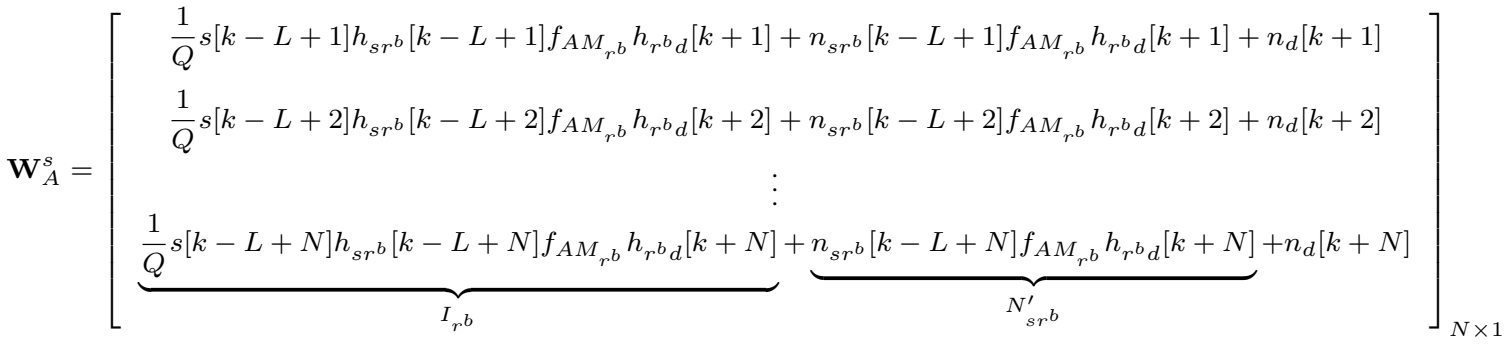

\begin{tabular}{|l|lll|}
\hline Benchmark-I & single-user scenario dispensing with relaying & non-coherent DQPSK detection employing MSDSD of [6] & $D F=0.01 N=6$ \\
\hline Benchmark-II & single-user scenario dispensing with relaying & coherent DQPSK detection & $D F=0.01$ \\
\hline Benchmark-III & single relay-aided two-phase cooperative scenario & non-coherent DQPSK detection employing MSDSD of [7] & $D F=0.02 N=6$ \\
\hline Benchmark-IV & single-user scenario dispensing with relaying & coherent DQPSK detection & $D F=0.06$ \\
\hline
\end{tabular}

TABLE I

DEFINITIONS OF BENCHMARKS EMPLOYED IN FIGURES 4 AND 5

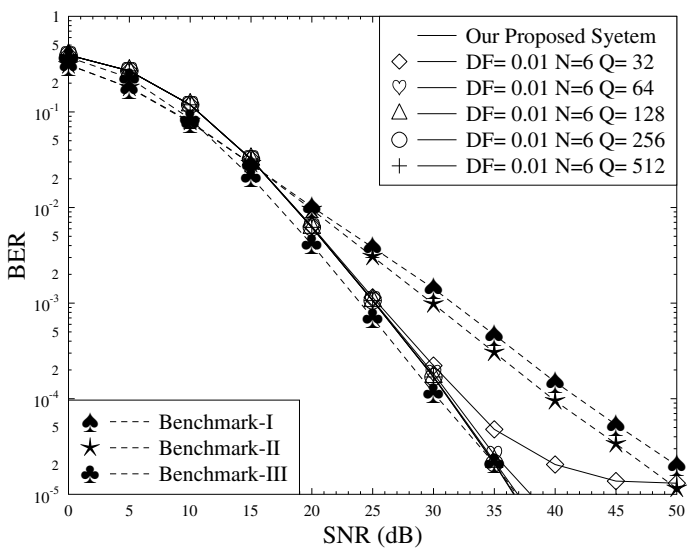

Fig. 4. BER versus SNR performance of our relay-aided MSDSD assisted successive relaying based cooperative DS-CDMA uplink configured with various SF values, when transmitting via time-selective Rayleigh fading channels. Three different benchmark curves are provided.

two-phase cooperative counterpart, which also employed MSDSD. The associated performance degradation is attributable to two main reasons: firstly, to the successive-relaying-induced interference between the transmitted signals of the $\mathrm{SN}$ and $\mathrm{RN}$; secondly, to the noise $N_{s r^{u}}^{\prime}$ amplified and forwarded by the $u$ th RN, which is imposed on the despread source signal, but would be eliminated in DAF cooperative communication. Nevertheless, our MSDSD assisted successive relaying aided system still substantially outperforms the conventional single-user schemes operating without RNs, which hence also eliminate the problem of throughput reduction. For example, observe in Figure 4 that our scheme results in a performance gain of about $10 \mathrm{~dB}$ at a target BER of $10^{-4}$ compared to the benchmark-I curve. It is also clearly seen in Figure 4 that our scheme maintains a performance gain of about $8 \mathrm{~dB}$ at a target BER of $10^{-4}$ compared to the conventional coherent single-user DQPSK scheme dispensing with relaying.

Apart from increasing the spatial diversity order for the sake of reducing the detrimental impact of time-selective fading channels, increasing the observation window size of the MSDSD algorithm is another beneficial method, which results in an improved time diversity. However, when the window size is extended, the complexity imposed increases rapidly. In order to assess the trade-offs between the attainable time diversity and the system's complexity, simulation results are provided in Figures 5 and 6. Other parameters, such as the normalized DF, the spreading factor and the modulation schemes, are fixed to $D F=0.06, S F=512$ and DQPSK, respectively.

As expected, an increased performance gain is attained, when the window size increases from $N=3$ to 12 , as shown in Figure 5. If the window size is relatively small, such as $N=3$ or 4 , the performance of the new system becomes slightly worse than that of the conventional single-user coherent DQPSK scheme dispensing with relaying with the same normalized DF of 0.06 , but gradually improves upon increasing $N$. However, no further significant performance gain may be attained, once the window size reached $N=8$, despite investing substantially increased search complexity, as quantified in terms of the number of multiplications required for decoding a single symbol. 


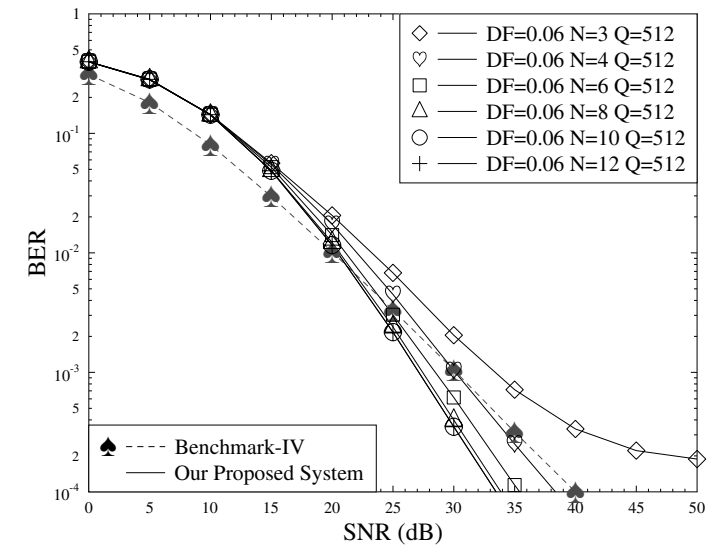

Fig. 5. The effect of the MSDSD observation window size on the BER performance of the relay-aided MSDSD assisted successive relaying based DS-CDMA uplink, when experiencing time-selective Rayleigh fading channels and a DF of 0.06 .

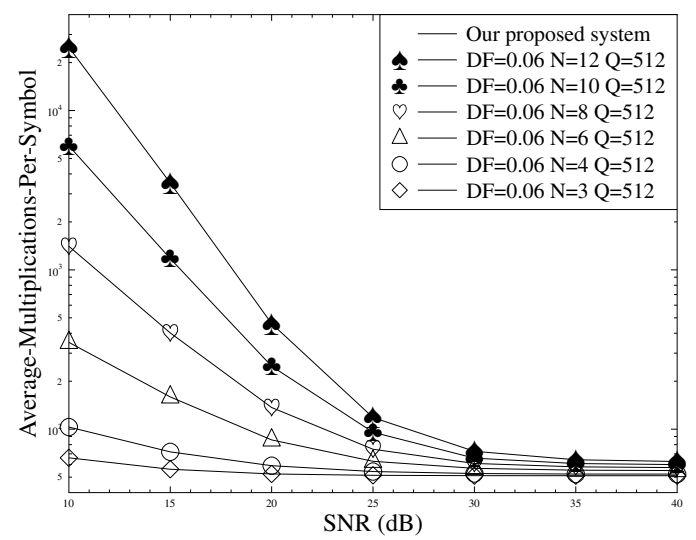

Fig. 6. Complexity versus SNR performance of the relay-aided MSDSD assisted successive relaying based cooperative DS-CDMA uplink with respect to various observation window sizes.

This is particularly so in the low-SNR region, as shown in Figure 6. In practical applications, the BER should be lower than $10^{-3}$. In other words, according to Figure 5, the new system performs well for SNRs above $30 \mathrm{~dB}$. In this SNR range of Figure 6, the complexity difference is no longer significant between the different window sizes. This justifies our previous statements, arguing that a window size of $N=8$ could be a meritorious choice, which has a moderate complexity, whilst achieving the best BER performance in the context of the parameters considered.

\section{CONCLUSIONS}

In this contribution, we have proposed a distributed relay-aided multiple-symbol differential sphere detection scheme designed for a successive relaying aided DS-CDMA system. The $50 \%$ throughput loss of the conventional two-phase single relay aided network is recovered by employing an additional relay. The challenges of mitigating the successive relaying-induced interference without relying on any channel state information were also successfully solved in the context of the classic DS-CDMA technique. The simulation results of Figure 4 demonstrated that our novel scheme maintains a significant performance gain of up to $10 \mathrm{~dB}$ over the benchmark schemes employed.

\section{REFERENCES}

[1] G. J. Foschini and M. J. Gans, "On Limits of Wireless Communications in a Fading Environment when Using Multiple Antennas," Wireless Personal Communications, vol. 6, pp. 311-335, Mar 1998.
[2] Van der Meulen, E.C., "Three-Terminal Communication Channels," Advanced Applied Probability, vol. 3, pp. 120-154, 1971.

[3] L. Hanzo, O. R. Alamri, M. El-Hajjar and N. Wu, "Near-Capacity Multi-Functional MIMO Systems: Sphere-Packing, Iterative Detection and Cooperation," John Wiley, May 2009.

[4] H. V. Trees, "Detection, Estimation and Modulation Theory: Part I," John Wiley, March 1968.

[5] P. Ho and D. Fung, "Error Performance of Multiple Symbol Differential Detection of PSK signals Transmitted over Correlated Rayleigh Fading Channels," IEEE Transactions on Communications, vol. 40, pp. 25-29, October 1992.

[6] L. Lampe, R. Schober, V. Pauli, and C. Windpassinger, "MultipleSymbol Differential Sphere Decoding," IEEE Transactions on Communications, vol. 12, pp. 1981-1985, Dec 2005.

[7] L. Wang and L. Hanzo, "The Amplify-and-Forward Cooperative Uplink Using Multiple-Symbol Differential Sphere-Detection,” IEEE Signal Processing Letters, vol. 16, pp. 913-916, October 2009.

[8] L. Hanzo, Y. Akhtman, M. Jiang and L. Wang, "MIMO-OFDM for LTE, WIFI and WIMAX: Coherent versus Non-Coherent and Cooperative Turbo-Transceivers," John Wiley, 2010.

[9] Y. Fan, C. Wang, J. Thompson, H. V. Poor, "Recovering Multiplexing Loss through Successive Relaying Using Repetition Coding," IEEE Transactions on Communications, vol. 6, pp. 4484-4493, December 2007.

[10] S. Won, K. Lee, and L. Hanzo, "Initial Code Acquisition in the Cooperative Noncoherent MIMO DS-CDMA Downlink," IEEE Transactions on Vehicular Technology, vol. 58, pp. 1387-1395, March 2009.

[11] L. Kong, S. X. Ng, R. G. Maunder, and L. Hanzo, "Near-Capacity Cooperative Space-Time Coding Employing Irregular Design and Successive Relaying," IEEE Transactions on Communications, vol. 58, pp. 22322241, August 2010.

[12] W. Fang, L.-L. Yang and L. Hanzo, "Single-User Performance of DirectSequence Code-Division Multiple-Access Using Relay Diversity and Power Allocation," IET Proceedings of Communications, vol. 2, pp. 462472, March 2008

[13] T. Himsoon, W. Su, and K. J. R. Liu, "Differential Transmission for Amplify-and-Forward Cooperative Communications," IEEE Signal Processing letters, vol. 12, pp. 597-600, September 2005.

[14] A. Abrardo, "Noncoherent MLSE Detection of M-DPSK for DS-CDMA Wireless Systems," IEEE Transactions on Vehicular Technology, vol. 52, pp. 1435-1446, November 2003. 